\title{
Manejo integral de los recortes de perforación de la industria petrolera en Tabasco Integrated management of drill cuttings the oil industry in Tabasco
}

\author{
René Méndez Villegas \\ Universidad Tecnológica de Tabasco \\ renemv68@hotmail.com \\ Petrona Gómez Rivera \\ Universidad Tecnológica de Tabasco \\ pegori11@hotmail.com \\ Juan Ismael Ledesma Herrera \\ Universidad Tecnológica de Tabasco \\ clasificado_spt@hotmail.com \\ Claudia María del Carmen Ceniceros González \\ Universidad Tecnológica de Tabasco \\ cenicerosclaudia1610@hotmail.com
}

\section{Resumen}

Los recortes de perforación impregnados con lodos base agua y de emulsión inversa base aceite, son considerados residuos de manejo especial de acuerdo a la Ley para la Prevención y Gestión Integral de los Residuos del Estado de Tabasco, publicada en 2013. Hay que señalar que el estado no contaba con un instrumento de regulación para estos residuos; sin embargo, como resultado del trabajo donde se propone el Manejo Integral de los Recortes de Perforación de la Industria Petrolera en Tabasco, se logró que se considerara dentro de la propuesta de la Ley citada, que afortunadamente fue publicada en el Periódico Oficial del Gobierno del Estado. Este trabajo establece los lineamientos para realizar un manejo integral, consistente en la generación, almacenamiento, transporte, reciclaje, coprocesamiento, tratamiento y disposición final de los recortes de perforación, ya que estos residuos son generados en grandes cantidades por la industria petrolera en Tabasco, especialmente en el proceso de perforación. 
Palabras clave: manejo integral, recortes de perforación, residuos de manejo especial, reusar, tratamiento.

\section{Abstract}

Cuts impregnated drilling muds water and oil-based invert emulsion are considered special waste management according to the Law for the Prevention and Management of Waste in Tabasco State, published in 2013. Should be noted that the state did not have a regulatory instrument for these wastes; however, as a result of work where the Integrated Management of drilling cuttings of the Oil Industry in Tabasco aims was achieved which was considered in the proposal of the Act, which fortunately was published in the Official Gazette of the Government of state. This paper provides guidelines for integrated management, involving the generation, storage, transportation, recycling, coprocessing, treatment and disposal of drill cuttings, as these wastes are generated in large quantities by the oil industry in Tabasco, especially in the drilling process.

Key words: integrated management, drill cuttings, special handling waste, refuse treatment.

Fecha recepción: Enero 2013

Fecha aceptación: Marzo 2013

\section{Introducción}

Los recortes de perforación de acuerdo a la NOM-115-SEMARNAT-2003, son los fragmentos de roca que se obtienen del proceso de perforación; constituidos por minerales de las formaciones perforadas, entre otros, arcillas, cuarzo, feldespatos, carbonatos y otros compuestos calcáreos y de sílice que están impregnados con fluidos de perforación; en la siguiente figura se muestran ejemplos de recortes de perforación.

Se tiene como base legal la entrada en vigor de la Ley General para la Prevención y Gestión Integral de los Residuos (LGPGIR) en el año 2003, donde se precisa la distribución de competencias a la federación, los estados y municipios, la regulación ambiental para el manejo de los residuos, tanto peligrosos, de manejo especial y sólidos urbanos. Corresponde a la federación regular los residuos peligrosos, a las entidades federativas los residuos de manejo especial y a los municipios los residuos sólidos urbanos. 
Dentro de la regulación de los residuos de manejo especial por parte de los estados, se contempla a los residuos de recortes de perforación impregnados con fluidos base agua y emulsión inversa base aceite generados por la industria petrolera, originados en la etapa de perforación de los pozos petroleros que se encuentran en el estado de Tabasco.

Para el año 2008, 334 mil toneladas de recortes de perforación derivados de las actividades de exploración y producción y lo generado en el activo integral Samaria-Luna, en el periodo enerodiciembre 2011, fue de 25,729.39 toneladas.

La Secretaría de Energía, Recursos Naturales y Protección Ambiental (SERNAPAM), trabaja en congruencia con el Plan Estatal de Desarrollo 2007-2012, que establece, en su eje transformador siete, la estrategia para el diseño e instrumentación de políticas y programas para el control y disminución de la contaminación del suelo, aire y agua, mediante la línea de acción para instrumentar el programa estatal de gestión de los residuos de manejo especial. Por tal sentido, requiere de mecanismos de evaluación y capacidades metodológicas para el manejo ambientalmente adecuado de los residuos de manejo especial, incluyendo la valoración de las tecnologías a utilizar.

Este trabajo se realizó en dos etapas, la primera etapa consistió en realizar un diagnóstico de generación y composición fisicoquímica de los recortes de perforación en la industria petrolera y la segunda etapa el realizar la propuesta de manejo de los recortes de perforación en congruencia con la Ley General para la Prevención y Gestión Integral de los Residuos y su reglamento, así como la Ley de Protección Ambiental de Estado de Tabasco y la Ley para la Prevención y Gestión Integral de los Residuos del Estado de Tabasco.

La propuesta de manejo contempla el almacenamiento adecuado en los sitios de generación y en sitios temporales de transferencia, el transporte de los recortes en equipos y medios de transportes como góndolas, pipas y volteos con todos los implementos de seguridad y los lineamientos establecidos por la Secretaría de Comunicaciones y Transportes, el tratamiento que se les puede aplicar a los recortes, de acuerdo a las autorizaciones emitidas a los procesos y empresas por parte de la autoridad ambiental estatal, el reciclaje y la disposición final. 


\section{DESARROLLO}

El trabajo se desarrolló en dos etapas, la primera etapa, correspondió el realizar el Foro de consulta denominado "Foro sobre Manejo de los Residuos de Perforación de la Industria Petrolera en Tabasco 2012", en las instalaciones de la Universidad Tecnológica de Tabasco, con la participación de 60 personas provenientes de 18 empresas que realizan o prestan el servicio a la industria petrolera, los cuales fueron distribuidos en cuatro mesas de análisis y discusión. Se habló de los temas de perforación y generación de recortes, tratamiento de los recortes de perforación, transporte de los recortes de perforación, normatividad y gestión de los recortes de perforación.

Posteriormente se realizaron visitas a cuatro instalaciones petroleras, específicamente pozos petroleros en perforación, ubicados en el municipio de Cunduacán, Tabasco. Estos recorridos comprendieron las áreas de perforación (equipos), área de almacenamiento de fluidos de perforación y de recortes de perforación, verificando la generación de los recortes de perforación base agua y base aceite empleado y la forma de manejo que se le aplica a los recortes de perforación, generando como producto el diagnóstico de generación de residuos de recortes de perforación impregnados con fluidos base agua y emulsión inversa base aceite originados por la industria petrolera considerados de manejo especial, el cual arrojó que la tasa de generación anual distribuida en el activo Samaria-Luna, en el periodo enero-diciembre 2011, fue de 25,729.39 toneladas.

Asimismo, se realizaron visitas a instalaciones de tratamiento de recortes de perforación, para conocer los procesos de tratamiento, el manejo que se les aplica a los residuos antes del tratamiento y su tratamiento, la infraestructura existente y los mecanismos de emergencia aplicados.

Para la segunda etapa, corresponde elaborar la propuesta de manejo integral de los recortes de perforación de la industria petrolera en el estado de Tabasco, considerando para ello los criterios de evaluación, validación y autorización de los protocolos de prueba de tecnologías para el manejo y tratamiento de los residuos de recortes de perforación impregnados con fluidos base agua y emulsión inversa base aceite originados por la industria petrolera considerados de manejo 
especial, los tipos de transportes utilizados, el sistema de almacenamiento temporal, así como la valorización y/o reutilización.

\section{IDENTIFICACIÓN DE LOS PROCESOS DONDE SE GENERAN LOS RECORTES DE PERFORACIÓN}

La perforación es un proceso que consiste en realizar en el subsuelo un hueco vertical, inclinado u horizontal, para alcanzar profundidades que van en promedio de 3 a 6 kilómetros de extensión con el objetivo de llegar a sitios conocidos como formaciones posiblemente productoras de hidrocarburos.

Para el proceso de perforación es indispensable la utilización de lodos o fluidos de perforación, en cuyo caso se utilizan lubricantes o lodos de perforación que son mezclas preparadas con gran cantidad de aditivos químicos.

Es importante resaltar que en la etapa de perforación se generan grandes cantidades de residuos sólidos o recortes de perforación. Si se tiene un pozo típico de 16,000 pies (aproximadamente 5 kilómetros) de profundidad pueden ser generados 4000 barriles de recortes de perforación, los cuales se componen principalmente de arenas, arcillas, minerales y aditivos. El mayor inconveniente en este caso no se produce por el volumen de sólidos que se generan, sino por la toxicidad de estos, ya que estuvieron en continuo contacto con el fluido de perforación, por lo que es muy común tener recortes con impregnaciones de aceites o cualquier otro contaminante.

El volumen de recortes generados depende de la profundidad y diámetro de la perforación. Los sólidos son continuamente removidos vía el equipo de control de sólidos. El mayor volumen se genera en las etapas iniciales donde el diámetro del agujero es mayor, la descarga puede ser continua e intermitente ocurriendo por periodos de menos de 1 y hasta 24 horas por día, dependiendo del tipo de operación y características del pozo.

\section{COMPOSICIÓN DE LOS RECORTES DE PERFORACIÓN}

La composición de los recortes de perforación base agua y base aceite, va relacionado con los tipos de hidrocarburos y compuestos orgánicos e inorgánicos, los cuales fueron analizados por un laboratorio acreditado por la Entidad Mexicana de Acreditación y se señalan a continuación. 
a) Recortes de perforación base agua

\begin{tabular}{|c|c|c|c|}
\hline PARÁMETRO & MÉTODO ANALÍTICO & UNIDADES & RESULTADO \\
\hline HIDROC. FRACC. PESADA & $\begin{array}{l}\text { EPA 1654A/9071B-1996 A3 } \\
\text { NOM-138-SEMARNAT/SS-2003 }\end{array}$ & $\mathrm{Mg} / \mathrm{Kg}$, B.S. & 54836.48 \\
\hline Humedad & AS-05 & $\%$ & 85.9 \\
\hline \multicolumn{4}{|l|}{ BTEX B.S. } \\
\hline Benceno & $\begin{array}{l}\text { EPA 8260-B 1995. NOM-138- } \\
\text { SEMARNAT/SS-2003 AN A4 }\end{array}$ & Ug/kg, B.S. & ND \\
\hline Tolueno & $\begin{array}{l}\text { EPA 8260-B } 1995 \text { NOM-138- } \\
\text { SEMARNAT/SS-2003 AN A4 }\end{array}$ & $\mathrm{Ug} / \mathrm{kg}$, B.S. & 12420.61 \\
\hline Etilbenceno & $\begin{array}{l}\text { EPA 8260-B } 1995 \text { NOM-138- } \\
\text { SEMARNAT/SS-2003 AN A4 }\end{array}$ & $\mathrm{Ug} / \mathrm{kg}$, B.S. & 26295.65 \\
\hline M,P-Xileno & $\begin{array}{l}\text { EPA 8260-B } 1995 \text { NOM-138- } \\
\text { SEMARNAT/SS-2003 AN A4 }\end{array}$ & $\mathrm{Ug} / \mathrm{kg}$, B.S. & 86380.50 \\
\hline O-Xileno & $\begin{array}{l}\text { EPA 8260-B } 1995 \text { NOM-138- } \\
\text { SEMARNAT/SS-2003 AN A4 }\end{array}$ & Ug/kg, B.S. & 54457.56 \\
\hline HIDROC. FRACC. MEDIA & $\begin{array}{l}\text { EPA 8015B-1996, NOM-138- } \\
\text { SEMARNAT/SS-2003 AN2 }\end{array}$ & $\mathrm{Mg} / \mathrm{Kg}, \mathrm{B} . \mathrm{S}$. & 138000.40 \\
\hline$>\mathrm{C} 10<\mathrm{C} 15$ & $\begin{array}{l}\text { EPA 8015B-1996, NOM-138- } \\
\text { SEMARNAT/SS-2003 AN2 }\end{array}$ & $\%$ & 45.33 \\
\hline$>\mathrm{C} 15<\mathrm{C} 20$ & $\begin{array}{l}\text { EPA 8015B-1996, NOM-138- } \\
\text { SEMARNAT/SS-2003 AN2 }\end{array}$ & $\%$ & 39.61 \\
\hline$>\mathrm{C} 20<\mathrm{C} 25$ & $\begin{array}{l}\text { EPA 8015B-1996, NOM-138- } \\
\text { SEMARNAT/SS-2003 AN2 }\end{array}$ & $\%$ & 12.05 \\
\hline$>\mathrm{C} 25<\mathrm{C} 28$ & $\begin{array}{l}\text { EPA 8015B-1996, NOM-138- } \\
\text { SEMARNAT/SS-2003 AN2 }\end{array}$ & $\%$ & 3.01 \\
\hline
\end{tabular}

\begin{tabular}{|l|l|c|c|}
\hline \multicolumn{1}{|c|}{ PARÁMETRO } & \multicolumn{1}{|c|}{ MÉTODO ANALÍTICO } & UNIDADES & RESULTADO \\
\hline HIDROC. FRACC. LIGERA & $\begin{array}{l}\text { EPA 1654A/9071B-1996 A1 } \\
\text { NOM-138-SEMARNAT/SS-2003 }\end{array}$ & $\mathrm{Mg} / \mathrm{Kg}, \mathrm{B} . \mathrm{S}$. & 79305.9 \\
\hline$<$ C5 & $\begin{array}{l}\text { EPA 8260-B 1995. NOM-138- } \\
\text { SEMARNAT/SS-2003 A1 }\end{array}$ & $\mathrm{Ug} / \mathrm{kg}, \mathrm{B} . \mathrm{S}$. & 0.005 \\
\hline$>$ C5<C10 & $\begin{array}{l}\text { EPA 8260-B 1995 NOM-138- } \\
\text { SEMARNAT/SS-2003 A1 }\end{array}$ & $\mathrm{Ug} / \mathrm{kg}, \mathrm{B} . \mathrm{S}$. & 99.994 \\
\hline $\begin{array}{l}\text { CONSTITUYENTES } \\
\text { INORGÁNICOS (METALES) }\end{array}$ & & & \\
\hline Arsénico & EPA 6010B-1996 & $\mathrm{Mg} / \mathrm{L}$ & 0.0169 \\
\hline Bario & EPA 6010B-1996 & $\mathrm{Mg} / \mathrm{L}$ & 1.6720 \\
\hline Cadmio & EPA 6010B-1996 & $\mathrm{Mg} / \mathrm{L}$ & 0.0117 \\
\hline Cromo & EPA 6010B-1996 & $\mathrm{Mg} / \mathrm{L}$ & $\mathrm{ND}$ \\
\hline Mercurio & EPA 7471A-1995 & $\mathrm{Mg} / \mathrm{L}$ & $\mathrm{ND}$ \\
\hline Plata & EPA 6010B-1996 & $\mathrm{Mg} / \mathrm{L}$ & $\mathrm{ND}$ \\
\hline Plomo & EPA 6010B-1996 & $\mathrm{Mg} / \mathrm{L}$ & 86180 \\
\hline Selenio & EPA 6010B-1996 & $\mathrm{Mg} / \mathrm{L}$ & 0.0223 \\
\hline
\end{tabular}

Tabla 1. Composición del recorte de perforación base agua. Fuente: Análisis cromatográfico realizado por laboratorio acreditado.

b) Recortes de perforación base aceite 


\begin{tabular}{|l|l|c|c|}
\hline \multicolumn{1}{|c|}{ PARÁMETRO } & \multicolumn{1}{|c|}{ MÉTODO ANALÍTICO } & UNIDADES & RESULTADO \\
\hline HIDROC. FRACC. PESADA & $\begin{array}{l}\text { EPA 1654A/9071B-1996 A3 } \\
\text { NOM-138-SEMARNAT/SS-2003 }\end{array}$ & $\mathrm{Mg} / \mathrm{Kg}, \mathrm{B} . \mathrm{S}$. & 54836.48 \\
\hline HIDROC. FRACC. MEDIA & $\begin{array}{l}\text { EPA 8015B-1996, NOM-138- } \\
\text { SEMARNAT/SS-2003 AN2 }\end{array}$ & $\mathrm{Mg} / \mathrm{Kg}, \mathrm{B} . \mathrm{S}$. & 138205.00 \\
\hline HIDROC. FRACC. LIGERA & $\begin{array}{l}\text { EPA 1654A/9071B-1996 A1 } \\
\text { NOM-138-SEMARNAT/SS-2003 }\end{array}$ & $\mathrm{Mg} / \mathrm{Kg}, \mathrm{B} . \mathrm{S}$. & 48.51 \\
\hline BTEX B.S. & & & \\
\hline Benceno & $\begin{array}{l}\text { EPA 8260-B 1995. NOM-138- } \\
\text { SEMARNAT/SS-2003 AN A4 }\end{array}$ & $\mathrm{Ug} / \mathrm{kg}, \mathrm{B} . \mathrm{S}$. & $\mathrm{ND}$ \\
\hline Tolueno & $\begin{array}{l}\text { EPA 8260-B 1995 NOM-138- } \\
\text { SEMARNAT/SS-2003 AN A4 }\end{array}$ & $\mathrm{Ug} / \mathrm{kg}, \mathrm{B} . \mathrm{S}$. & $\mathrm{ND}$ \\
\hline Etilbenceno & $\begin{array}{l}\text { EPA 8260-B 1995 NOM-138- } \\
\text { SEMARNAT/SS-2003 AN A4 }\end{array}$ & $\mathrm{Ug} / \mathrm{kg}, \mathrm{B} . \mathrm{S}$. & $\mathrm{ND}$ \\
\hline M,P-Xileno & $\begin{array}{l}\text { EPA 8260-B 1995 NOM-138- } \\
\text { SEMARNAT/SS-2003 AN A4 }\end{array}$ & $\mathrm{Ug} / \mathrm{kg}, \mathrm{B} . \mathrm{S}$. & $\mathrm{ND}$ \\
\hline O-Xileno & $\begin{array}{l}\text { EPA 8260-B 1995 NOM-138- } \\
\text { SEMARNAT/SS-2003 AN A4 }\end{array}$ & $\mathrm{Ug} / \mathrm{kg}, \mathrm{B} . \mathrm{S}$. & 0.19635 \\
\hline $\begin{array}{l}\text { CONSTITUYENTES } \\
\text { INORGÁNICOS (METALES) }\end{array}$ & & & \\
\hline Arsénico & EPA 6010B-1996 & $\mathrm{Mg} / \mathrm{L}$ & $\mathrm{ND}$ \\
\hline Bario & EPA 6010B-1996 & $\mathrm{Mg} / \mathrm{L}$ & 41140 \\
\hline Cadmio & EPA 6010B-1996 & $\mathrm{Mg} / \mathrm{L}$ & $\mathrm{ND}$ \\
\hline Cromo & EPA 6010B-1996 & $\mathrm{Mg} / \mathrm{L}$ & 0.0002 \\
\hline Mercurio & EPA 7471A-1995 & $\mathrm{Mg} / \mathrm{L}$ & $\mathrm{ND}$ \\
\hline Plata & EPA 6010B-1996 & 34830 \\
\hline Plomo & EPA 6010B-1996 & $\mathrm{Mg} / \mathrm{L}$ & \\
\hline Selenio & EPA 6010B-1996 & \\
\hline
\end{tabular}

Tabla 2. Composición del recorte de perforación base aceite. Fuente: Análisis cromatográfico realizado por laboratorio acreditado.

\section{MANEJO INTEGRAL}

\section{a) Almacenamiento}

Los recortes y residuos producidos durante la perforación con fluidos base agua se deben depositar en un confinamiento de terracería de 45 × 20 × 2 m, impermeabilizado y techado. De igual manera, los recortes producidos durante la perforación con fluido de emulsión inversa, se deberán depositar en un confinamiento de concreto de 20 × 15 × 2 m, impermeabilizado y techado.

Otra práctica común de almacenamiento es la presa de desperdicios, la cual debe ser construida de terracería, siguiendo los procedimientos de compactación requeridos para evitar infiltraciones al subsuelo, otras de ellas cuentan con geomembranas en las paredes para evitar este problema. Las presas tienen unas dimensiones de aproximadamente $20 \times 20 \times 1 \mathrm{~m}$, donde se acumulan los 
recortes de perforación impregnados con fluidos base agua. Una vez terminadas las operaciones de perforación, el fluido almacenado en las presas, denominado lodo limpio por ser base agua, es transportado a otras presas.

\section{b) Transporte}

Para el trasporte de recortes de perforación vía terrestre se deben cumplir los siguientes parámetros.

El transportista responsable del traslado de los recortes debe continuar con el control documental y físico sobre el manejo de los recortes.

Por ningún motivo debe excederse la velocidad de $10 \mathrm{~km} / \mathrm{h}$ dentro de las instalaciones petroleras. Para el tráfico fuera de instalaciones petroleras, debe darse cumplimiento a los límites de velocidad establecidos en la NOM-EM-033-SCT-2-2002 y las disposiciones establecidas por las autoridades locales, según corresponda.

En caso de que el prestador de servicios o contratista ocasione daños a terceros, este debe efectuar los pagos e indemnizaciones procedentes.

No se permite realizar labores de mantenimiento al transporte en instalaciones petroleras.

El prestador de servicios o contratista que realice el transporte terrestre de los recortes deben contar con el plan de contingencias aprobado por la dependencia ambiental en el estado de Tabasco.

El prestador de servicios o contratista del transporte terrestre, debe reunir los requisitos que establezca la normatividad vigente en materia ambiental y contar con las autorizaciones o permisos vigentes solicitados por la Secretaría de Comunicaciones y Transportes, o la autoridad competente según sea el caso.

El prestador de servicios o contratista debe contar con operadores para el transporte de recortes que reúnan los conocimientos técnicos y normativos en materia de seguridad industrial y manejo de residuos. 
En México, el marco jurídico ambiental que define la regulación en materia de transporte de residuos de manejo especial, está conformado por la Ley General para la Prevención y Gestión Integral de los Residuos y su Reglamento, al igual que el Reglamento para el Transporte Terrestre de Materiales y Residuos Peligrosos.

Como el trasporte de residuos de manejo especial es una actividad en la cual la Secretaría de Energía, Recursos Naturales y Protección Ambiental y la SCT tienen competencia, la vinculación por parte de estas dos autoridades ha permitido una evolución y mejora en la integralidad del control del proceso de transporte, a través de la expedición del permiso específico para esta actividad.

Lo anterior y otros requisitos adicionales se encuentran establecidos claramente en la guía de referencia para Trasporte de los Residuos Peligrosos de la SEMARNAT, debido a que el estado no cuenta con normatividad en la materia, como se menciona a continuación:

I. Expedición de permiso para la operación y explotación de los servicios de autotransporte federal de carga especializada de materiales, residuos, remanentes y residuos de manejo especial para la SCT y para la SERNAPAM.

II. Tal como lo establece el artículo 80 de la LGPGIR, las personas interesadas en obtener autorizaciones para llevar a cabo los servicios a terceros para el transporte, acopio, almacenamiento, reutilización, reciclaje, tratamiento y disposición final de residuos, según sea el caso, deberán presentar ante la Secretaría su solicitud de autorización (SEMARNAT-07-033-I), este documento deberá de contener la siguiente información.

III. Manifiesto de registro de actividades de manejo de residuos peligrosos, el cual tiene como finalidad asegurar que los RP designados para ser recolectados, transportados y entregados en los sitios externos para su tratamiento, almacenamiento y disposición final lleguen íntegros a su destino, debido a que en este formato se especifican las características del residuo y sus puntos de carga y entrega; además de constituir una guía de manejo, por parte del personal, en caso de algún incidente o accidente.

IV. Modificación a los registros y autorizaciones en material de residuo peligroso en los rubros de actualización de datos en registros y autorizaciones, aviso de modificación a la autorización 
por cambio de denominación o razón social o para transferencia de autorizaciones en materia de residuos peligrosos.

V. Aviso de derrame, infiltración, descarga o vertido de materiales peligrosos o residuos peligrosos:

En la modalidad de Aviso Inmediato (PROFEPA-03-017-A), que tiene como principal objetivo conocer inmediatamente de un peligro para el medio ambiente por parte de los responsables del manejo de materiales o residuos peligrosos.

VI. Expedición de permiso para la operación y explotación de los servicios de autotransporte federal de carga especializada de materiales, residuos, remanentes y desechos peligrosos tanto para personas morales como físicas (SCT-03-040-A/SCT-03-040-B).

VII. Expedición de permiso para la operación y explotación de los servicios de transporte privado de carga especializada de materiales, residuos, remanentes y desechos peligrosos tanto para personas morales como físicas (SCT-03-044-A/SCT-03-044-B).

La recolección de los lodos y recortes de perforación se realiza mediante el uso de góndolas y pipas de presión y vacío. En la siguiente tabla se muestran las características, capacidades y medidas de seguridad de cada uno de los equipos de recolección. 


\begin{tabular}{|c|c|c|c|}
\hline $\begin{array}{l}\text { Tipo de equipo } \\
\text { de recolección }\end{array}$ & Descripción & Capacidad & Medidas de seguridad \\
\hline Góndola & $\begin{array}{l}\text { - Largo } 9 \text { m, ancho } 2.60 \mathrm{~m} \text { y alto } \\
\text { - } 2 \mathrm{~m} . \\
\text { - } \text { Dos ejes } 30,000 \text { lbs. } \\
\text { - } \text { Suspensión neumática } \\
\text { - Porta llanta. } \\
\text { - Dos patines de velocidades } \\
\text { - Ocho llantas r-24.5 y rin r-24.5 } \\
\text { - Gancho de arrastre } \\
\text { - Luces reglamentarias } \\
\text { - Equipo hidráulico pistón } \\
\text { - telescópico } \\
\text { - } \text { Cinco secciones con extensión } \\
\text { - Rompe ola }\end{array}$ & $\begin{array}{l}30 \mathrm{~m}^{3} \mathrm{y} / \mathrm{o} \\
25 \text { toneladas }\end{array}$ & $\begin{array}{l}\text { Colocación de } \\
\text { geomembrana para } \\
\text { evitar derrames. } \\
\text { Tornillos de seguridad } \\
\text { en la parte trasera } \\
\text { para evitar } \\
\text { escurrimientos. }\end{array}$ \\
\hline $\begin{array}{l}\text { Pipa de presión y } \\
\text { vacío }\end{array}$ & $\begin{array}{l}\text { - Tanque cilíndrico } \\
\text { - Acero al carbón }\end{array}$ & 30,000 litros & $\begin{array}{l}\text { Dos válvulas de } \\
\text { esferas de } 3 \text { pulgadas, } \\
\text { parte trasera con } \\
\text { charola. } \\
\text { Válvula de } 2 \\
\text { pulgadas. }\end{array}$ \\
\hline
\end{tabular}

Tabla 3. Características de los equipos de recolección de lodos y recortes de perforación.

\section{c) Reutilización}

El recorte de perforación son pequeños trozos que se generan por la acción de rompimiento de la barrena en su penetración en la corteza terrestre, los cuales son arrastrados hacia la superficie por el fluido. El volumen de recortes generados depende de la profundidad y diámetro de perforación.

Los sólidos son continuamente removidos vía el equipo de control de sólidos. El mayor volumen se genera en las etapas iniciales, donde el diámetro del agujero es mayor, la descarga puede ser continua e intermitente ocurriendo por periodos de menos de 1 y hasta 24 horas por día, dependiendo del tipo de operación y características del pozo. Así, el total de sólidos puede ser por lo menos igual al volumen del agujero y algunas veces mayor, sin embargo, el sólido separado 
suele ser menor ya que una importante cantidad de ellos queda disperso en el fluido. Respecto a sus características, el recorte por sí solo es un sólido inerte, sin embargo, los sólidos de la perforación pueden contener restos de fluido adherido a ellos. La composición o presencia de contaminantes va a depender del fluido usado. Así los recortes asociados al uso de fluidos base aceite, podrán presentar contenido de hidrocarburos. Manteniendo una alta eficiencia del equipo de control de sólidos, es posible alcanzar una concentración de sólidos de hasta $96 \%$, siendo el restante $4 \%$ fluido de perforación adherido.

Existen diferentes tipos de alternativas de reutilización para los recortes de perforación, a continuación se mencionan algunas de ellas.

\section{Revestimiento en carreteras}

Este residuo puede ser usado como mezcla de revestimiento de carreteras, que posea características de densidad de mezcla y contenido de metales consistentes con las aprobadas para asfalto de carreteras o mezclas. En aplicaciones directas, a cargo del mismo generador en tramos de carreteras públicas o privadas, debe ser en relaciones de cargas que reduzcan la posibilidad de escurrimientos superficiales y apegados a las regulaciones locales. El reglamento que debe de observarse es el referente a mezclas de asfalto y materiales para la construcción de carreteras de la Secretaría de Comunicaciones y Transportes.

Sin embargo, existe otros materiales dentro de las actividades de perforación considerados como residuos y los cuales pueden ser reutilizados, tales como:

\section{d) Reciclaje}

Resulta importante recordar que, a pesar de que el reciclado ayuda a conservar recursos y reduce desechos, existen costos económicos y ambientales asociados con los procesos de recolección y reciclado. Es por ello que solo se debe considerar el reciclado para el caso de desechos que no pueden ser reducidos ni vueltos a utilizar.

Los desechos de una compañía pueden ser materia prima de otra compañía. 
Reciclar un desecho significa que se ha utilizado el desecho completo o que "va hacia" el proceso de reciclado.

El reciclado puede ser una medida doblemente beneficiosa.

La compañía que elimina el desecho ahorra el costo del transporte y disposición del desecho, mientras que el usuario ahorra en los costos de las materias primas.

Esta alternativa involucra la conversión del residuo a material utilizable y a la extracción /recuperación de energía o materiales de valor de los mismos. El reciclaje ayuda a conservar las riquezas y a reducir los desperdicios, es importante saber que hay gastos económicos y ambientales que están asociados con la recolección de los desperdicios y los procesos de recirculación. Por este motivo, el reciclaje debe ser considerado solamente para desperdicios que no puedan ser reducidos o vueltos a usar en el mismo proceso. Los residuos de estas actividades, pueden ser en un momento dado la materia prima a utilizar en otras actividades.

\section{Coprocesamiento}

Una alternativa para el uso posterior de los recortes de perforación sin tratar o mediante el tratamiento por algunas de las tecnologías aprobadas es el coprocesamiento para la producción de cemento. En el estado de Tabasco se cuenta con una planta productora de cemento que recibe estos tipos de residuos; además, por la distancia la hace atractiva y económica.

\section{Tratamientos}

Por lo general, el área ambiental debe de mantener información detallada de los "pros" y "contras" de cada tipo de tratamiento factible de aplicar, así como de las regulaciones aplicables vigentes, ya que con base en ellos se determinan los riesgos y costos y la forma de eliminar el residuo. Debe tomarse en cuenta que solo después de haber analizado la información anterior será conveniente llevar a cabo una evaluación de la tecnología de tratamiento. 
Los cinco principales tipos de procesamiento de residuos que están interrelacionados con los residuos son:

- Tratamiento físico. Procesos en el que mediante concentración y/o cambio de fase se modifican los constituyentes peligrosos a una forma más conveniente para su manejo posterior.

- Tratamiento químico. Procesos en donde los constituyentes peligrosos son modificados mediante reacciones químicas. En algunos casos equivale a una neutralización del peligro; en otros casos excepcionales, el residuo podrá seguir siendo peligroso pero en una forma más apropiada para su posterior manejo.

- Tratamiento biológico. Las modificaciones o diminución de la concentración se realizan mediante la acción de bacterias propias del suelo, y se aplica tanto para residuos peligrosos como no peligrosos.

- Tratamiento térmico. Proceso en el que se usa alta temperatura para la destrucción de tóxicos, principalmente orgánicos.

En el estado de Tabasco la Secretaría de Energía, Recursos Naturales y Protección Ambiental (SERNAPAM), autorizó algunas tecnologías para el tratamiento de los recortes de perforación base agua y base aceite de la industria petrolera, las cuales se mencionan a continuación:

\begin{tabular}{|l|l|}
\hline \multicolumn{1}{|c|}{ TECNOLOGÍAS } & \multicolumn{1}{|c|}{ TIPO DE PROCESO } \\
\hline $\begin{array}{l}\text { Tratamiento "in situ" u "onsite" de } \\
\text { residuos de manejo especial (lodos y recortes } \\
\text { de perforación impregnados con fluido base } \\
\text { agua y base aceite) mediante la } \\
\text { centrifugación y oxidación química. }\end{array}$ & - Proceso físico \\
\hline $\begin{array}{l}\text { b) Tratamiento de residuos de manejo } \\
\text { especial (lodos y recortes de perforación con } \\
\text { fluidos base aceite) por medio de la } \\
\text { tecnología de desestabilización fisicoquímica. }\end{array}$ & - Proceso físico \\
\hline $\begin{array}{l}\text { c) Tratamiento de residuos de manejo } \\
\text { especial (lodos y recortes de perforación con } \\
\text { fluidos base agua y base aceite) por medio de } \\
\text { la técnica de estabilización química. }\end{array}$ & - Proceso químico \\
\hline $\begin{array}{l}\text { d) Tratamiento de residuos de manejo } \\
\text { especial (lodos y recortes de perforación con }\end{array}$ & - Proceso químico \\
\hline
\end{tabular}




\begin{tabular}{|c|c|}
\hline $\begin{array}{l}\text { fluidos base agua y base aceite) por medio de } \\
\text { la técnica de oxidación química. }\end{array}$ & \\
\hline $\begin{array}{l}\text { e) Tratamiento de residuos de manejo } \\
\text { especial (lodos y recortes de perforación } \\
\text { impregnados con fluidos base agua y base } \\
\text { aceite, tierra y materiales semejantes a suelo } \\
\text { contaminados y/o impregnados por lodos y } \\
\text { recortes de perforación con fluidos base agua } \\
\text { y base aceite) mediante el proceso de } \\
\text { degradación de contaminantes mediante las } \\
\text { fases física-oxidación, química-degradación } \\
\text { bioquímica, aplicada en tres modalidades de } \\
\text { tratamiento "Ex Situ", "OnSite” e "In Situ”. }\end{array}$ & $\begin{array}{ll}\text { - } & \text { Proceso físico } \\
\text { - } & \text { Proceso químico } \\
\text { - } & \text { Proceso biológico }\end{array}$ \\
\hline $\begin{array}{l}\text { f) Tratamiento “in situ” u "onsite" de } \\
\text { residuos de manejo especial (lodos y recortes } \\
\text { de perforación impregnados con fluido base } \\
\text { agua y base aceite) mediante la degradación } \\
\text { bioquímica }\end{array}$ & $\begin{array}{l}\text { - Proceso químico } \\
\text { - Proceso biológico }\end{array}$ \\
\hline $\begin{array}{l}\text { g) Tratamiento ex situ de residuos de } \\
\text { manejo especial (lodos y recortes de } \\
\text { perforación con fluidos base agua, base } \\
\text { aceite, lodos provenientes del tratamiento de } \\
\text { aguas aceitosas y sanitarias) mediante la } \\
\text { biorremediación y lavado de suelos. }\end{array}$ & $\begin{array}{l}\text { - } \text { Proceso físico } \\
\text { - } \quad \text { Proceso biológico }\end{array}$ \\
\hline $\begin{array}{l}\text { h) Tratamiento residuos de manejo } \\
\text { especial (lodos y recortes de perforación con } \\
\text { fluidos base agua) mediante métodos físico, } \\
\text { químico y biológico. }\end{array}$ & $\begin{array}{l}\text { - Proceso físico } \\
\text { - Proceso químico } \\
\text { - Proceso biológico }\end{array}$ \\
\hline $\begin{array}{l}\text { i) Tratamiento de residuos de manejo } \\
\text { especial (lodos y recortes de perforación } \\
\text { impregnados con fluido base aceite) por } \\
\text { medio del proceso de desorción química } \\
\text { (oxidación química y destilación por arrastre } \\
\text { de vapor), }\end{array}$ & $\begin{array}{l}\text { - Proceso químico } \\
\text { - Proceso térmico }\end{array}$ \\
\hline $\begin{array}{l}\text { j) Tratamiento de residuos de manejo } \\
\text { especial (lodos y recortes de perforación con } \\
\text { fluidos base agua) por medio de la técnica de } \\
\text { lavado. }\end{array}$ & - Proceso físico \\
\hline $\begin{array}{l}\text { k) Tratamiento ex situ de residuos de } \\
\text { manejo especial (lodos } y \text { recortes de }\end{array}$ & - Proceso químico \\
\hline
\end{tabular}




perforación impregnados con fluido base
agua) por medio del proceso de
deshidratación.

1) Tratamiento ex situ de residuos de manejo especial (recortes de perforación impregnados con fluidos base agua y base aceite, lodos sedimentados resultantes del tratamiento de aguas residuales sanitarias y lodos sedimentados del tratamiento de aguas industriales aceitosas) mediante la tecnología de lavado de suelos y degradación biológica.

m) Tratamiento de residuos de manejo especial in situ y ex situ (lodos y recortes de perforación impregnados con fluidos base agua, base aceite y suelos contaminados con

- Proceso químico estos residuos) por medio de la técnica de bioestimulación/degradación.

n) Tratamiento ex situ e in situ de residuos de manejo especial (lodos y recortes de perforación con fluidos base agua y base aceite), suelos contaminados con recortes base agua y base aceite.

o) Tratamiento de residuos de manejo especial (lodos y recortes de perforación impregnados con fluido base agua) por medio de las técnicas de oxidación química y desenmulsificación.

Tabla 4. Tratamientos autorizados por la SERNAPAM, para los recortes de perforación en el estado de Tabasco. Fuente: Análisis de la aplicabilidad potencial de las tecnologías para el tratamiento de los residuos de manejo especial a las condiciones específicas del Estado de Tabasco. (Villegas, 2013)

\section{e) Disposición final}

De la información recopilada es posible establecer cuatro procedimientos de disposición final, los cuales son:

- Disposición sobre terreno como material de relleno.

- Inyección de recortes.

- Depósito en confinamiento controlado. 
- Cierre adecuado de las presas durante las actividades de abandono.

En su aplicación deberán tomarse en cuenta, entre otras, las consideraciones siguientes:

- $\quad$ La disposición debe desarrollarse de una manera prudente y responsable.

- $\quad$ El volumen de bombeo de la fase líquida de las presas debe ser controlada para que el líquido no se escurra por lugares que no hayan sido destinados para su eliminación.

- La disposición no debe producir erosión. La tierra utilizada para la eliminación no puede ser tan empinada ni tener tan poca vegetación que no pueda contener el flujo desenfrenado de líquido. El terreno debe ser nivelado.

- $\quad$ No se deben usar aguas superficiales para la eliminación.

- El riesgo de contaminación de corrientes de aguas subterráneas debe ser mínimo. Esto imposibilita la eliminación sobre tierra granular.

- Debe evitarse la tierra de poco espesor como un suelo esquelético sobre un lecho de roca.

\section{Disposición sobre terreno como material de relleno}

Esta opción requiere de tratamiento previos como; concentración de sólidos/deshidratación, solidificación/estabilización y probablemente de solidificación.

En la zona sur se ha llevado a la práctica el tratamiento para su disposición final de 3,009 ton de recortes, mediante el proceso de encapsulamiento, el cual es realizado por conducto de una compañía especializada. Hasta el primer semestre de 1994, se habían tratado mediante este proceso aproximadamente 600 ton. Los recortes encapsulados fueron donados al Ayuntamiento de Paraíso, Tabasco, para ser usados como material de relleno, previa liberación por parte de la Secretaría del Medio Ambiente y Recursos Naturales (antes SEDESOL).

\section{Confinamiento controlado}

La disposición de sólidos, recortes de perforación en confinamiento controlado, constituye una alternativa muchas veces recomendable. Esta forma de disposición es la que se encuentra mejor desarrollada con base en criterios de ingeniería. El confinamiento controlado son instalaciones para el depósito de residuos en forma controlada, clasificada y duradera. Los residuos en la 
mayoría de los casos requieren previamente de tratamiento fisicoquímico y de incineración (detoxificación, deshidratación, reducción de volumen).

Para no causar efectos adversos al medio ambiente, los confinamientos deben realizarse bajo los siguientes criterios:

1) Cuidadosa selección del sitio

2) Construcción, operación y criterios según lineamientos

3) Recubrimiento apropiado

4) Cuidados después del recubrimiento

5) Monitoreo de agua subterránea y lixiviados, y

6) Registro de datos.

A un confinamiento no pueden enviarse lodos o recortes de perforación, si estos presentan líquidos libres que puedan separarse de la porción sólida bajo condiciones normales. Es necesario que los residuos sean previamente: deshidratados, detoxificados y neutralizados, mediante procesos de tratamiento físicos y químicos, cumpliendo además con las limitaciones que regulan la operación de un confinamiento.

\section{CONCLUSIONES}

En el estado de Tabasco, la industria petrolera ha estado y sigue realizando estudios de prospección para el descubrimiento de nuevos yacimientos petroleros y ha continuado con la perforación de pozos en los diferentes activos de producción en la entidad. Para ello se utiliza una gran cantidad de lodos o fluidos de perforación que permite lubricar las barrenas para que estas no se deterioren o atasquen y se tengan pérdidas considerables en la operación.

Los recortes de perforación son producidos en la etapa donde los fluidos se mezclan con los remantes rocosos y de suelo y emergen a través de las tuberías hacia el exterior, que son recepcionados mediante la descarga a tanques de almacenamiento temporal. Una parte de los lodos es recuperada al igual que los recortes y el resto son enviados a través de equipo de transporte diseñado para tal fin en góndolas o pipas de presión y vacío. 
De acuerdo a lo establecido por la norma oficial mexicana NOM-052-SEMARNAT-2005, se señala que los recortes de perforación no son considerados residuos peligrosos, por lo que de acuerdo a la Ley General para la Prevención y Gestión Integral de los Residuos, los clasifica como residuos de manejo especial. De igual manera, la Ley para la Prevención y Gestión Integral de los Residuos del Estado de Tabasco, lo considera como un residuo de manejo especial, por lo que le corresponde a la entidad regular su manejo.

En ese sentido, se llevó a cabo el Foro sobre Manejo de los Residuos de Perforación de la Industria Petrolera en Tabasco 2012, para conocer la situación actual de manejo que se le realiza a los recortes de perforación, participando también dependencias de gobierno en materia ambiental, la industria petrolera, consultoras ambientales, empresas de servicios para la industria petrolera e instituciones educativas y de investigación.

Asimismo, se realizó un diagnóstico de generación y manejo de los recortes de perforación, tomando como caso de estudio el activo integral Jujo-Reforma, dos instalaciones petroleras y dos empresas de servicios de tratamiento de los recortes de perforación ubicadas en el estado de Tabasco.

Con esas premisas, se diseñó la propuesta de Manejo Integral de los Recortes de Perforación de la industria petrolera en Tabasco, para que la dependencia normativa lo considerara como parte de la gestión de la paraestatal y de todas aquellas empresas que le den servicios a Petróleos Mexicanos y que involucren a estos residuos. Esta propuesta considera los elementos mínimos necesarios para cumplir con la normatividad ambiental estatal en aspectos de almacenamiento, transporte, reutilización, reciclaje, tratamiento y disposición final.

Además, se logró que la normativa considerara estos aspectos en la Ley de Prevención y Gestión Integral de los Residuos del Estado de Tabasco y estableciera el Protocolo de Pruebas para los tratamientos autorizados como términos de referencia y homologación. 


\section{Bibliografía}

LAGREGA, Michael D., Phillip, L., Buckingham, J. (1996). Gestión de Residuos Toxicas (Tratamiento, Eliminación y Recuperación de Suelos). España, Madrid: McGraw Hill Interamericana de España.

PAZ, Miguel A. (2013). Memoria de estadía Profesional. Clasificación y Diagnóstico del Manejo de los Residuos de Recortes de Perforación con Fluido de Emulsión Inversa Base Aceite de la Industria Petrolera. Universidad Tecnológica de Tabasco.119 Pag.

SÁNCHEZ, Pérez J. (1996). Aspectos básicos de geología para la biorremediación de suelos. Curso de biorremediación de suelos y acuíferos. PUMA, UNAM.

SERNAPAM (2012). Ley para la Prevención y Gestión Integral de los Residuos del Estado de Tabasco. Periódico Oficial del Gobierno del Estado de Tabasco. 7 de diciembre de 2012.

SERNAPAM (2012). Ley de Protección Ambiental del Estado de Tabasco. Periódico Oficial del Gobierno del Estado de Tabasco. 5 de diciembre de 2012.

SEMARNAT (2013). Ley General para la Prevención y Gestión Integral de los Residuos. Diario Oficial de la Federación. 5 de noviembre de 2013.

SEMARNAT (2008). Las 3Rs en el programa nacional para la prevención y gestión integral de los residuos 2009-2012. Ciudad de México, octubre 2008.

SOBERANEZ, Iván T. (2013). Memoria de estadía Profesional. Clasificación y Diagnóstico del Manejo de los Residuos de Recortes de Perforación con Fluido Base Aguae de la Industria Petrolera. Universidad Tecnológica de Tabasco.114 Pag. 